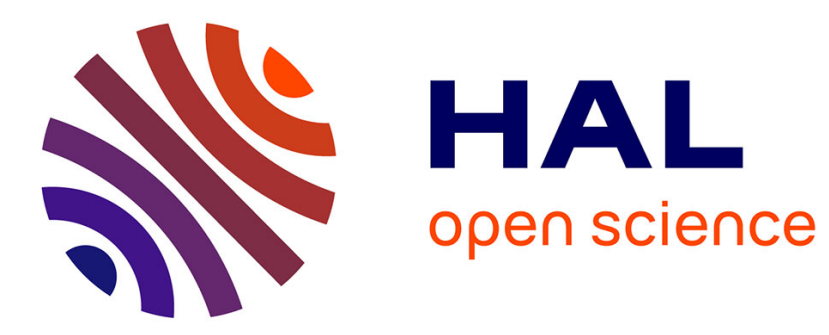

\title{
Generalized Luenberger observers for fault detection in switched systems using $\mathbf{H}$ - index
}

\author{
Ahmad Farhat, Damien Koenig
}

\section{To cite this version:}

Ahmad Farhat, Damien Koenig. Generalized Luenberger observers for fault detection in switched systems using H- index. ECC 2016 - 15th European Control Conference, Jun 2016, Aalborg, Denmark. hal-01372951

\section{HAL Id: hal-01372951 \\ https://hal.science/hal-01372951}

Submitted on 27 Sep 2016

HAL is a multi-disciplinary open access archive for the deposit and dissemination of scientific research documents, whether they are published or not. The documents may come from teaching and research institutions in France or abroad, or from public or private research centers.
L'archive ouverte pluridisciplinaire HAL, est destinée au dépôt et à la diffusion de documents scientifiques de niveau recherche, publiés ou non, émanant des établissements d'enseignement et de recherche français ou étrangers, des laboratoires publics ou privés. 


\title{
Generalized Luenberger observers for fault detection in switched systems using $\mathrm{H}$ - index
}

\author{
Ahmad Farhat ${ }^{1}$ and Damien Koenig ${ }^{1}$
}

\begin{abstract}
In this paper, a linear matrix inequality (LMI) technique for the fault detection and state estimation of switched discrete systems is proposed. This approach is employed to generate generalized Luenberger minimal order observers giving best state estimation and sensitive fault detection. It consists on using $H_{-}$index design. Sufficient conditions of global convergence of observers are proposed using switched Lyapunov functions. Numerical example is given to illustrate this method.

Keywords : Fault detection, reduced order observers, switched systems, LMI, $H_{-}$.
\end{abstract}

\section{INTRODUCTION}

Model based techniques have been widely studied and developed for fault detection (FD) problems [1]-[4].

On another hand, switched system modeling has been of considerable interest, since many physical plants show switching feature between sub-models, where multicontrollers/observer with suitable switching rules are needed [5], [6].

The classical observers for FD consist on generating the residual signal. In literature, numerous methods use LMIs techniques, such as $H_{\infty}$ approach, the sensitivity of fault approach, $H_{\infty} / H_{-}$approach [7]-[9].

Only few works have extended the $H_{-}$fault detection technique by adding weighting filters (analogue to the standard $H_{\infty}$ control problem) [8], [10]. It greatly improves the fault sensitivity specially when the spectral frequency of the faults is known (zeros- frequencies for offset or Boolean faults, low frequencies for low varying faults...) .

In this paper the $H_{-}$approach is adopted, coupled with a minimal order observer strategy. The motivation for the reduced order state observer stems from the fact that only $p$ of $n$ states are directly available from measurement, and only the other $n-p$ states are unavailable, hence it suffices to build an observer that estimates at least the unmeasured states. Numerous technique have been studied based on algebraic equation from generalized Luenberger observers [11], [12].

The main contributions of this paper are:

- To give sufficient conditions for existence of generalized Luenberger minimal order observer for state estimation and fault detection.

- To give a general scheme to insure fault sensitivity, by solving an optimization problem using LMIs formulated by multi-objectives $H_{-} /$stability design.

\footnotetext{
${ }^{1}$ A. Farhat and D. Koenig are with Univ. Grenoble Alpes, GIPSA-lab, F-38000 Grenoble, France. (ahmad.farhategipsa-lab.grenoble-inp.fr, damien.koenigdesisar.grenoble-inp.fr)
}

To the best of our knowledge, the problem of the minimal order observers design for state estimation and fault detection for switched systems has not been investigated yet. The $H_{-}$ switched observer design uses switched Lyapunov functions (SLF) in order to avoid conservatism introduced by one common Lyapunov function.

The outline of this paper is as follows. After the introduction, general structure of the observer is given in Section II. In section III, preliminaries and sufficient conditions on the existence and parametrization of the observer are stated. The synthesis is discussed in Section IV with the use of LMI to satisfy $H_{-}$and stability objectives. A general design scheme is given in Section V, then followed by results illustrated in a numerical example in Section VI. Finally, the last section shows the concluding remarks and the possible future work.

Notations: The notation used in this paper is standard. $X^{T}$ is the transposed of matrix $X$, the star symbol $(\star)$ in a symmetric matrix denotes the transposed block in the symmetric position; The notation $P>(<, \leq) 0$ means $P$ is real symmetric positive (negative, semi) definite matrix; $A^{+}$ is the generalized inverse matrix of $A$ satisfying $A A^{+} A=A$; 0 and $I$ denote zeros and identity matrix of appropriate dimensions.

\section{PRoblem Formulation}

Consider the following class of linear discrete-time switched system with faults and disturbances:

$$
\left\{\begin{array}{cl}
x_{k+1} & =A_{\alpha(k)} x_{k}+B_{\alpha(k)} u_{k}+E_{f, \alpha(k)} f_{k} \\
y_{k} & =C_{\alpha(k)} x_{k}+F_{f, \alpha(k)} f_{k}
\end{array}\right.
$$

where $x \in \mathbb{R}^{n}, y \in \mathbb{R}^{p}, u \in \mathbb{R}^{m}$ and $f \in \mathbb{R}^{n_{f}}$ denote respectively the state vector, the measurement output vector, the input vector and the vector of faults to be detected.

The switching rule takes values in the finite index set $\mathbb{S}=$ $\left\{1,2, . ., N_{\alpha}\right\}$. It means that the switched signal is a piecewise constant function, where $N_{\alpha}$ is the number of subsystems. When $\alpha(k)=i$, it means that the $i^{t h}$ subsystem is activated. Moreover, at the switching time $k: i=\alpha(k) \neq \alpha(k+1)=j$. Then (1) can be transformed to:

$$
\left\{\begin{array}{cl}
x_{k+1} & =A_{i} x_{k}+B_{i} u_{k}+E_{f, i} f_{k} \\
y_{k} & =C_{i} x_{k}+F_{f, i} f_{k}
\end{array}\right.
$$

The aim is to design a generalized observer in the form:

$$
\left\{\begin{aligned}
z_{k+1} & =F_{i} z_{k}+G_{i} y_{k}+J_{i} u_{k} \\
\hat{x}_{k} & =M_{i} z_{k}+N_{i} y_{k}
\end{aligned}\right.
$$


where $z \in \mathbb{R}^{q}$ is the state vector of the observer, $\hat{x} \in \mathbb{R}^{n}$ is the estimation of $x$, Matrices $F_{i}, G_{i}, J_{i}, M_{i}$ and $N_{i}$ are unknown to be determined and of appropriate dimensions.

In order to find the unknown matrices, an error variable is introduced: $\epsilon_{k}=z_{k}-T_{i} x_{k}$, where $T_{i} \in \mathbb{R}^{q \times n}$ is an arbitrary matrix.

Remark 1: Assume that $\operatorname{rank}\left(C_{i}\right)=p$, if $q=n-p$, a minimal order observer is obtained; if $q=n$, a reduced order observer is obtained, the full-order observer is obtained if $n-p<q<n$. The minimal order observer problem is investigated in this study.

Assumptions: In the sequel it is assumed that:

A. 1: The pairs $\left(A_{i}, C_{i}\right)$ are observable, or without loss of generality are detectable. This is a standard for all fault detection problems in the free unknown inputs (IU) case. This could be written as:

$$
\operatorname{rank}\left(\begin{array}{c}
z I_{n}-A_{i} \\
C_{i}
\end{array}\right)=n
$$

A. 2: $\operatorname{rank}\left(\begin{array}{l}T_{i} \\ C_{i}\end{array}\right)=n$, and that there exist matrices $E_{i} \in$ $\mathbb{R}^{q \times n}$ such that:

$$
\operatorname{rank}\left(\begin{array}{c}
E_{i} \\
C_{i}
\end{array}\right)=\operatorname{rank}\left(\begin{array}{c}
T_{i} \\
C_{i}
\end{array}\right)=n
$$

A. 3: The switching signal $\alpha(t)$ is not known a priori, but it is measured and its value is real time available.

A. 4: Faults occur in low frequencies, it is the region where most faults are spectraly located (offsets...).

\section{OBSERVER PARAMETRIZATION}

Lemma 1: For $f_{k}=0$, (3) is a generalized observer for system (1) if there exists arbitrary matrices $T_{i}$ and $T_{j}$ such that (s.t.) the following constraints are satisfied:

$$
\begin{array}{r}
F_{i} T_{i}+G_{i} C_{i}-T_{j} A_{i}=0 \\
J_{i}-T_{j} B_{i}=0 \\
M_{i} T_{i}+N_{i} C_{i}=I_{n}
\end{array}
$$

and the matrices $F_{i}$ are Hurwitz.

Proof 1: By definition, the state estimate is:

$$
\hat{x}_{k}=M_{i} z_{k}+N_{i} y_{k}=M_{i} \epsilon_{k}+\left(M_{i} T_{i}+N_{i} C_{i}\right) x_{k}
$$

If $\epsilon_{k} \rightarrow 0$ then $\hat{x}_{k} \rightarrow x_{k}$; it follows

$$
\hat{x}_{k}=M_{i} \epsilon_{k}+x_{k}
$$

which is true if (6c) holds.

Secondly, from the definition of the error $\epsilon$ :

$$
\begin{aligned}
\epsilon_{k+1} & =z_{k+1}-T_{j} x_{k+1} \\
& =F_{i} z_{k}+G_{i} y_{k}+J_{i} u_{k}-T_{j} A_{i} x_{k}-T_{j} B_{i} u_{k} \\
& =F_{i} \epsilon_{k}+\left(F_{i} T_{i}+G_{i} C_{i}-T_{j} A_{i}\right) x_{k}+\left(J_{i}-T_{j} B_{i}\right) u_{k}
\end{aligned}
$$

The dynamics of the error is independent of $x$ and $u$ if the constraints (6a)-(6b) are satisfied.
Now, if the constraints in (6a)-(6c) are fulfilled, and defining the estimation error $e=x-\hat{x}$, the following autonomous system is deduced:

$$
\left\{\begin{array}{cc}
\epsilon_{k+1} & =F_{i} \epsilon_{k} \\
e_{k} & =M_{i} \epsilon_{k}
\end{array}\right.
$$

It is then obvious that the estimation error $e_{k} \rightarrow 0$ if $\epsilon_{k} \rightarrow$ 0 , and $\epsilon_{k} \rightarrow 0$ if and only if (iff) $F_{i}$ is Hurwitz.

In this part, the observer design problem in the fault/disturbance free case is reduced to the stabilization problem in (10).

The equation (6c) can be put on the form:

$$
\left[\begin{array}{ll}
M_{i} & N_{i}
\end{array}\right]\left[\begin{array}{l}
T_{i} \\
C_{i}
\end{array}\right]=I_{n}
$$

which has a solution according to [13] iff :

$$
\operatorname{rank}\left(\begin{array}{c}
T_{i} \\
C_{i} \\
I_{n}
\end{array}\right)=\operatorname{rank}\left(\begin{array}{c}
T_{i} \\
C_{i}
\end{array}\right)=n
$$

Since $T_{i}$ are unknown, there always exists matrices $T_{i}$ and $K_{i}$ such that:

$$
\begin{gathered}
{\left[\begin{array}{l}
T_{i} \\
C_{i}
\end{array}\right]=\left[\begin{array}{cc}
I_{q} & -K_{i} \\
0 & I_{p}
\end{array}\right]\left[\begin{array}{c}
E_{i} \\
C_{i}
\end{array}\right]} \\
\Longleftrightarrow \quad T_{i}=E_{i}-K_{i} C_{i} \\
\Longleftrightarrow\left[\begin{array}{ll}
T_{i} & K_{i}
\end{array}\right]\left[\begin{array}{l}
I_{n} \\
C_{i}
\end{array}\right]=E_{i}
\end{gathered}
$$

Again, (13) has a solution iff [13] :

$$
\operatorname{rank}\left(\begin{array}{c}
I_{n} \\
C_{i} \\
E_{i}
\end{array}\right)=\operatorname{rank}\left(\begin{array}{c}
I_{n} \\
C_{i}
\end{array}\right)=n
$$

Under (A.2), (12) and (14) are satisfied, then one solution for $T_{i}$ and $K_{i}$ is:

$$
T_{i}=E_{i}\left[\begin{array}{l}
I_{n} \\
C_{i}
\end{array}\right]^{+}\left[\begin{array}{c}
I_{n} \\
0
\end{array}\right] \text { and } K_{i}=E_{i}\left[\begin{array}{l}
I_{n} \\
C_{i}
\end{array}\right]^{+}\left[\begin{array}{c}
0 \\
I_{p}
\end{array}\right]
$$

And (11) becomes:

$$
\left[\begin{array}{ll}
M_{i} & N_{i}
\end{array}\right]\left[\begin{array}{cc}
I_{q} & -K_{i} \\
0 & I_{p}
\end{array}\right]\left[\begin{array}{l}
E_{i} \\
C_{i}
\end{array}\right]=I_{n}
$$

Denote:

$$
\begin{cases}\Gamma_{i}=\left[\begin{array}{c}
E_{i} \\
C_{i}
\end{array}\right], \quad \Gamma_{i}^{\perp}=\left[I_{p+q}-\Gamma_{i} \Gamma_{i}^{+}\right], \\
\Pi_{1, i}=\left[\begin{array}{c}
I_{q} \\
0
\end{array}\right], \quad \Pi_{2, i}=\left[\begin{array}{c}
K_{i} \\
I_{p}
\end{array}\right], \quad \Pi_{3, i}=\left[\begin{array}{c}
0 \\
I_{p}
\end{array}\right]\end{cases}
$$

Then (16) has the generalized solution using the pseudoinverse [13]:

$$
\left[\begin{array}{ll}
M_{i} & N_{i}
\end{array}\right]=\left(\Gamma_{i}^{+}-L_{1, i} \Gamma_{i}^{\perp}\right)\left[\begin{array}{cc}
I_{q} & K_{i} \\
0 & I_{p}
\end{array}\right]
$$

or equivalently:

$$
\begin{aligned}
M_{i} & =\Gamma_{i}^{+} \Pi_{1, i}-L_{1, i} \Gamma_{i}^{\perp} \Pi_{1, i}, \\
N_{i} & =\Gamma_{i}^{+} \Pi_{2, i}-L_{1, i} \Gamma_{i}^{\perp} \Pi_{2, i}
\end{aligned}
$$


where $L_{1, i}$ is arbitrary matrix.

Next, in order to compute $F_{i}$ and $G_{i}$, equation (6a) can be put in the form using (13b):

$$
\begin{array}{r}
F_{i}\left(E_{i}-K_{i} C_{i}\right)+G_{i} C_{i}=T_{j} A_{i} \\
\Longleftrightarrow \quad\left[\begin{array}{ll}
F_{i} & G_{i}-F_{i} K_{i}
\end{array}\right] \Gamma_{i}=T_{j} A_{i}
\end{array}
$$

This has a solution if the rank condition is satisfied [13]:

$$
\operatorname{rank}\left(\begin{array}{c}
\Gamma_{i} \\
T_{j} A_{i}
\end{array}\right)=\operatorname{rank}\left(\Gamma_{i}\right)=\operatorname{rank}\left(\begin{array}{c}
E_{i} \\
C_{i}
\end{array}\right)=n
$$

Which is true under (A.2). Then a generalized solution for the above equation is:

$$
\left[\begin{array}{ll}
F_{i} & G_{i}-F_{i} K_{i}
\end{array}\right]=T_{j} A_{i} \Gamma_{i}^{+}-L_{2, i} \Gamma_{i}^{\perp}
$$

Or equivalently:

$$
\begin{aligned}
F_{i} & =T_{j} A_{i} \Gamma_{i}^{+} \Pi_{1, i}-L_{2, i} \Gamma_{i}^{\perp} \Pi_{1, i}, \\
G_{i} & =T_{j} A_{i} \Gamma_{i}^{+} \Pi_{3, i}-L_{2, i} \Gamma_{i}^{\perp} \Pi_{3, i}+F_{i} K_{i} \\
& =T_{j} A_{i} \Gamma_{i}^{+}\left(\Pi_{3, i}+\Pi_{1, i} K_{i}\right)-L_{2, i} \Gamma_{i}^{\perp}\left(\Pi_{3, i}+\Pi_{1, i} K_{i}\right) \\
& =T_{j} A_{i} \Gamma_{i}^{+} \Pi_{2, i}-L_{2, i} \Gamma_{i}^{\perp} \Pi_{2, i}
\end{aligned}
$$

Denote:

$$
\begin{array}{ll}
\mathbb{A}_{1, i}=T_{j} A_{i} \Gamma_{i}^{+} \Pi_{1, i}, & \mathbb{A}_{2, i}=\Gamma_{i}^{\perp} \Pi_{1, i} \\
\mathbb{G}_{1, i}=T_{j} A_{i} \Gamma_{i}^{+} \Pi_{2, i}, & \mathbb{G}_{2, i}=\Gamma_{i}^{\perp} \Pi_{2, i}
\end{array}
$$

Then the autonomous system (10) becomes:

$$
\left\{\begin{array}{ccc}
\epsilon_{k+1} & = & \left(\mathbb{A}_{1, i}-L_{2, i} \mathbb{A}_{2, i}\right) \epsilon_{k} \\
e_{k} & = & M_{i} \epsilon_{k}
\end{array}\right.
$$

Lemma 2: The pair $\left(A_{i}, C_{i}\right)$ is detectable is equivalent to the detectability of the pair $\left(\mathbb{A}_{1, i}, \mathbb{A}_{2, i}\right)$.

Proof 2: See appendix

Theorem 1: Considering the system (25), necessary and sufficient condition to the stability of (25) is to find matrices $U_{i}$ and positive symmetric matrices $P_{i}$ and $P_{j}, \forall i, j \in \mathbb{S}$ satisfying:

$$
\left[\begin{array}{cc}
-P_{i} & \mathbb{A}_{1, i}^{T} P_{j}+\mathbb{A}_{2, i}^{T} U_{j}^{T} \\
P_{j} \mathbb{A}_{1, i}+U_{i} \mathbb{A}_{2, i} & -P_{j}
\end{array}\right]<0
$$

where $L_{2, i}=-P_{j}^{-1} U_{j}$ is a stabilizing gain.

Proof 3: Consider the Switched Lyapunov Functions (SLF) $V_{\alpha(k)}=\epsilon_{k}^{T} P_{\alpha(k)} \epsilon_{k}$, where $P_{\alpha(k)}>0$ is a positive definite matrix. If such a Lyapunov function exists, and its difference $\Delta V=V_{\alpha(k+1)}-V_{\alpha(k)}$ is negative definite along system trajectories of (25), then the origin of the system (25) is globally asymptotically stable. By computing the difference $\Delta V$ along the solution of (25), $\Delta V$ is given by:

$$
\begin{gathered}
\Delta V=\epsilon_{k+1}^{T} P_{\alpha(k+1)} \epsilon_{k+1}-\epsilon_{k}^{T} P_{\alpha(k)} \epsilon_{k}<0 \\
\Leftrightarrow \epsilon_{k}^{T}\left(\mathbb{A}_{1, \alpha(k)}-L_{2, \alpha(k)} \mathbb{A}_{2, \alpha(k)}\right)^{T} P_{\alpha(k+1)}\left(\mathbb{A}_{1, \alpha(k)}\right. \\
\left.-L_{2, \alpha(k)} \mathbb{A}_{2, \alpha(k)}\right) \epsilon_{k}-\epsilon_{k}^{T} P_{\alpha(k)} \epsilon_{k}<0
\end{gathered}
$$

That holds $\forall \epsilon_{k} \neq 0$, and by Schur complement with $U_{\alpha(k)}=$ $L_{2, \alpha(k)} P_{\alpha}(k)$ :

$$
\left[\begin{array}{cc}
-P_{\alpha(k)} & \mathbb{A}_{1, \alpha(k)}^{T} P_{\alpha(k+1)}-\mathbb{A}_{2, \alpha(k)}^{T} U_{\alpha(k+1)}^{T} \\
\star & -P_{\alpha(k+1)}
\end{array}\right]<0
$$

As this inequality has to be satisfied under the arbitrary switching law, it follows that it should hold for special configuration $\alpha(k+1)=j$ and $\alpha(k)=i, \forall i, j \in \mathbb{S}$.

Remark 2: Switched Lyapunov Functions (SLF) are used to avoid the conservatism generated when using a unique Lyapunov function for all sub-models. For each state $i$, different values of $P_{i}$ and $L_{2, i}$ are calculated. The condition on the edges between consecutive switches is guaranteed by the inclusion of $P_{j}$ in the LMIs.

To summarize, the algorithm for the proposed observer in fault/disturbances free case is:

1) Choose free matrices $E_{i}$ according to (5);

2) Compute $T_{i}$ and $K_{i}$ from (15) ;

3) Compute $\Gamma_{i}^{+}, \Gamma_{i}^{\perp}, \Pi_{1, i}, \Pi_{2, i}, \Pi_{3, i}$, from (17), then $N_{i}$, $M_{i}$ and $J_{i}$ from (19) and (6b);

4) Compute the gain $L_{2, i}$ solution of (26);

5) Deduce the matrices $F_{i}$ and $G_{i}$ according to (23a) and (23b).

In the following section, the step 4) is detailed and a procedure to compute the gain $L_{2, i}$ is given such that the designed observer meets the $H_{-} /$stability performances.

Remark 3: Since $L_{1, i}$ in (19) have no affect the system's dynamics, they could be chosen equal to 0 . This simplifies the calculations without altering the optimization problem in the following sections.

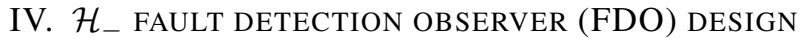

In this section, the observer design problem is studied as a fault detection problem. The aim of the observer design is to find a residual signal $r_{k}$ that is sensitive to faults at some rate $\beta_{i}$. This is expressed by:

$$
\left\|r_{i}\right\|_{2}>\beta_{i}\|f\|_{2}
$$

The objective of the $\mathcal{H}_{-}$switched FD observer is resumed by the following condition:

$$
\left\|T_{r f_{i}}\right\|_{-}>\beta_{i}
$$

where $T_{r f_{i}}(z)$ denotes the transfer function of the residual sensitivities faults .

Now when considering $f_{k} \neq 0$, and using the same constraints in (6a),(6c), while substituting (6b) by:

$$
J_{i}-T_{i} B_{i}=0
$$

The state estimation becomes:

$$
\hat{x}_{k}=M_{i} \epsilon_{k}+x_{k}+N_{i} F_{f, i} f_{k}
$$

The residuals signal that is defined by $r=y-\hat{y}$ becomes:

$$
\begin{aligned}
r_{k} & =C_{i} x_{k}+F_{f, i} f_{k}-\left(C_{i} \hat{x}_{k}\right) \\
& =C_{i}\left(x_{k}-\hat{x}_{k}\right)+F_{f, i} f_{k} \\
& =C_{i} M_{i} \epsilon_{k}+\left(C_{i} N_{i}+I\right) F_{f, i} f_{k}
\end{aligned}
$$

And the dynamic of the error variable $\epsilon$ becomes:

$$
\epsilon_{k+1}=F_{i} \epsilon_{k}+\left(G_{i} F_{f, i}-T_{i} E_{f, i}\right) f_{k}
$$


Then the residuals generator has the following state space representation:

$$
\left\{\begin{array}{l}
\epsilon_{k+1}=F_{i} \epsilon_{k}+\left(G_{i} F_{f, i}-T_{i} E_{f, i}\right) f_{k} \\
r_{k}=C_{i} M_{i} \epsilon_{k}+\left(C_{i} N_{i}+I\right) F_{f, i} f_{k}
\end{array}\right.
$$

It can be put in the generic form:

$$
\left\{\begin{array}{l}
\epsilon_{k+1}=\mathbb{A}_{i} \epsilon_{k}+\mathbb{B}_{f, i} f_{k} \\
r_{k}=\mathbb{C}_{i} \epsilon_{k}+\mathbb{D}_{f, i} f_{k}
\end{array}\right.
$$

where:

$$
\left\{\begin{array}{l}
\mathbb{A}_{i}=\mathbb{A}_{1, i}-\mathbb{L}_{i} \mathbb{A}_{2, i}, \quad \mathbb{B}_{f, i}=\mathbb{B}_{1 f, i}-\mathbb{L}_{i} \mathbb{B}_{2 f, i} \\
\mathbb{B}_{1 f, i}=\mathbb{G}_{1, i} F_{f, i}-T_{i} E_{f, i}, \quad \mathbb{B}_{2 f, i}=\mathbb{G}_{2, i} F_{f, i} \\
\mathbb{C}_{i}=C_{i} M_{i}, \quad \mathbb{D}_{f, i}=\left(C_{i} N_{i}+I\right) F_{f, i}, \quad \mathbb{L}_{i}=L_{2, i}
\end{array}\right.
$$

\section{A. $\mathcal{H}_{-}$fault sensitivity observer design}

The $\mathcal{H}_{-}$observer design problem in the presence of faults is expressed as an optimization problem by finding the gain matrix $\mathbb{L}_{i}$ such that the $\mathcal{H}_{-}$norm $\left\|T_{r f_{i}}\right\|_{-}$is maximized subject to $\beta_{i}$

Theorem 2: For a given switched LTI system with faults as defined in (37), there exist gain vectors $\mathbb{L}_{i}$ that satisfy the $\mathcal{H}_{-}$ observer design problem if there exits for $i, j \in \mathbb{S}$, symmetric positive matrices $\mathbb{P}_{i}$ and $\mathbb{P}_{j}$, matrices $\mathbb{U}_{i}$ and positive scalars $\beta_{i}$, such that the following LMIs are satisfied:

$$
\left[\begin{array}{ccc}
-\mathbb{P}_{j}-\mathbb{C}_{i}^{T} \mathbb{C}_{i} & -\mathbb{C}_{i}^{T} \mathbb{D}_{d, i} & \mathbb{A}_{1, i}^{T} \mathbb{P}_{i}+\mathbb{A}_{2, i}^{T} \mathbb{U}_{i} \\
\star & -\mathbb{D}_{f, i}^{T} \mathbb{D}_{f, i}+\beta_{i}^{2} I & \mathbb{B}_{1, i}^{T} \mathbb{P}_{i}+\mathbb{B}_{2, i}^{T} \mathbb{U}_{i} \\
\star & \star & -\mathbb{P}_{i}
\end{array}\right]<0
$$

Proof 4: Consider the switched Lyapunov function $V_{k}=$ $\epsilon_{k}^{T} P_{\alpha(k)} \epsilon_{k}$ where $P_{\alpha(k)}>0$. If such a function exist, and the following equality hold, then the The $\mathcal{H}_{-}$fault sensitivity is guaranteed.

$$
\epsilon_{k+1}^{T} P_{\alpha(k+1)} \epsilon_{k+1}-\epsilon_{k}^{T} P_{\alpha(k)} \epsilon_{k}-r_{k}^{T} r_{k}+\beta_{i}^{2} f_{k}^{T} f_{k}<0
$$

The rest of the proof is omitted here for lack of space, where steps of calculations are very similar to the bounded real lemma.

\section{B. $\mathcal{H}_{-}$synthesis problem}

As it has been proposed in [14], if $\mathbb{D}_{f, i}=0$ then $\mathcal{H}_{-}$ index is always zero, and the LMI (39) is infeasible (diagonal element is positive), and for $\mathbb{D}_{f, i} \neq 0$, the optimization problem would give $\beta_{i}=\left\|\mathbb{D}_{f, i}\right\|$

To avoid this restriction, solutions have been presented in [14] and [10], it consists on adding fictive weighting filters to the design: by adding an auxiliary direct channel to the system, and then multiply by a high pass filter $\mathcal{F}_{H}$ as it is shown in figure (1)

For $\mathbb{D}_{f, i}=\left[\begin{array}{c}\mathbb{D}_{1 f, i} \\ 0\end{array}\right]$, a suitable matrix is $\mathbb{D}_{a d d, i}=\left[\begin{array}{c}0 \\ \epsilon_{i} I\end{array}\right]$.

The high pass filter $\mathcal{F}_{H}$ is a weighting filter that is used to raise up the high-frequency response, so that minimum

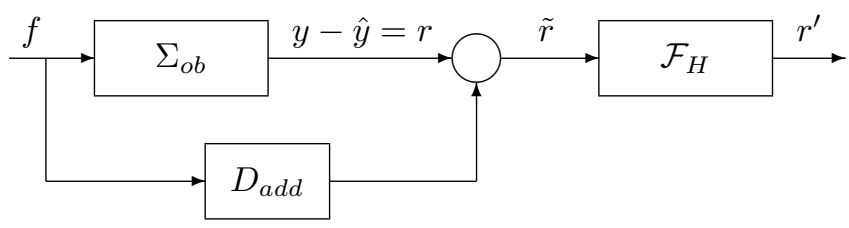

Fig. 1. $\mathcal{H}_{-}$Loop Shaping with Additive filter weighting

singular value of the whole system occurs near the lowfrequency region. $\mathcal{F}_{H}$ has the following transfer function:

$$
F_{H, i}(s)=\left(\frac{s / \omega_{1, i}+1}{s / \omega_{2, i}+1}\right)^{m_{i}}
$$

where $\omega_{1, i}<\omega_{2, i}$ and $m_{i}$ the order of the filter.

The parameters $\omega_{1, i}, \omega_{2, i}$ and $m_{i}$ are chosen such that the transfer function $T_{r f_{i}}(s)$ has the desired shape. This procedure is analog to the loop shaping method in the standard $\mathcal{H}_{\infty}$ problem.

The observer with the additive filter becomes:

$$
\left\{\begin{array}{l}
\epsilon_{k+1}=\mathbb{A}_{i} \epsilon_{k}+\mathbb{B}_{d, i} w_{k}+\mathbb{B}_{f, i} f_{k} \\
r_{k}=y_{k}-\hat{y}_{k}=\mathbb{C}_{i} \epsilon_{k}+\mathbb{D}_{d, i} w_{k}+\mathbb{D}_{f, i} f_{k} \\
\tilde{r}_{k}=(y-\hat{y})+\mathbb{D}_{a d d, i} f \\
x_{k+1}^{h}=A_{h, i} x_{k}^{h}+B_{h, i} \tilde{r}_{k} \\
r_{k}^{\prime}=C_{h, i} x_{k}^{h}+D_{h, i} \tilde{r}_{k}
\end{array}\right.
$$

where $F_{H, i}\left(q^{-1}\right):=\left[\begin{array}{c|c}A_{h, i} & B_{h, i} \\ \hline C_{h, i} & D_{h, i}\end{array}\right]$ is a discrete time realization of the high pass filter $\mathcal{F}_{H_{i}}$.

From equations (42), an augmented residual is deduced:

$$
\left\{\begin{aligned}
{\left[\begin{array}{c}
\epsilon_{k+1} \\
x_{k+1}^{h}
\end{array}\right]=} & {\left[\begin{array}{cc}
\mathbb{A}_{1, i}-L_{i} \mathbb{A}_{2, i} & 0 \\
B_{h, i} \mathbb{C}_{i} & A_{h, i}
\end{array}\right]\left[\begin{array}{c}
\epsilon_{k} \\
x_{k}^{h}
\end{array}\right] } \\
& +\left[\begin{array}{c}
\mathbb{B}_{1 f, i}-L_{i} \mathbb{B}_{2 f, i} \\
B_{h, i}\left(\mathbb{D}_{f, i}+\mathbb{D}_{a d d, i}\right)
\end{array}\right] f_{k} \\
r_{k}^{\prime} & {\left[\begin{array}{ll}
D_{h_{i}} \mathbb{A}_{2, i} & C_{h_{i}}
\end{array}\right]\left[\begin{array}{c}
\epsilon_{k} \\
x_{k}^{h}
\end{array}\right] } \\
& +\left[D_{h, i}\left(\mathbb{D}_{f, i}+\mathbb{D}_{a d d, i}\right)\right] f_{k}
\end{aligned}\right.
$$

Theorem (2) is then applied to (43), which yields to the following theorem:

Theorem 3: The $\mathcal{H}_{-} /$stable FDO for the augmented system in (43) is guaranteed, if there exist for $i, j \in \mathbb{S}$ and given positive reel scalars $\beta_{i}$, matrices $U_{a_{i}}$ and symmetric positive matrices $P_{i}^{a}$ and $P_{j}^{a}$ s.t. the following LMIs hold:

$$
\begin{gathered}
{\left[\begin{array}{c|c|c}
-P_{j}^{a}-C_{a_{i}}^{T} C_{a_{i}} & -C_{a_{i}}^{T} D_{a f_{i}} & A_{0_{i}}^{T} P_{i}^{a}+C_{0_{i}}^{T} U_{a_{i}}^{T} \\
\hline \star & -D_{a f_{i}}^{T} D_{a f_{i}} & B_{f 0_{i}}^{T} P_{i}^{a} \\
+\beta_{i}^{2} I & +F_{f 0_{i}}^{T} U_{a_{i}}^{T} \\
\hline \star & \star & -P_{i}^{a}
\end{array}\right]<0} \\
{\left[\begin{array}{ccc}
-P_{j}^{a} & 0 & A_{0_{i}}^{T} P_{i}^{a}+C_{0_{i}}^{T} U_{a_{i}}^{T} \\
\star & 0 & B_{f 0_{i}}^{T} P_{i}^{a}+F_{f 0_{i}}^{T} U_{a_{i}}^{T} \\
\star & \star & -P_{i}^{a}
\end{array}\right] \leq 0}
\end{gathered}
$$


where the filters gain are $L_{i}=I_{0}^{T} P_{a_{i}}^{-1} U_{a_{i}}$, and the matrices $A_{0_{i}}, B_{f 0_{i}}, C_{0_{i}}, F_{f 0_{i}}$ and $I_{0}$ are:

$$
\begin{array}{ll}
A_{0_{i}}=\left[\begin{array}{cc}
\mathbb{A}_{1, i} & 0 \\
B_{h, i} \mathbb{C}_{i} & A_{h, i}
\end{array}\right], I_{0}=\left[\begin{array}{c}
-I \\
0
\end{array}\right], & C_{0_{i}}=\left[\begin{array}{ll}
\mathbb{A}_{2, i} & 0
\end{array}\right], \\
B_{f 0_{i}}=\left[\begin{array}{cc}
\mathbb{B}_{1 f, i} \\
B_{h, i}\left(\mathbb{D}_{f, i}+\mathbb{D}_{a d d, i}\right)
\end{array}\right], & F_{f 0_{i}}=\left[\begin{array}{ll}
\mathbb{B}_{2 f, i} & 0
\end{array}\right]
\end{array}
$$

Proof 5: Let $x_{k}^{a}$ be the augmented state of the augmented system, $x_{k}^{a}=\left[\begin{array}{ll}\epsilon_{k}^{T} & x_{k}^{h T}\end{array}\right]^{T}$. The corresponding augmented switched Lyapunov function $V_{k}^{a}=\epsilon_{k}^{T} P_{\alpha(k)}^{a} \epsilon_{k}$ is considered. The $\mathcal{H}_{-}$objective for the FDO design are guaranteed by the inequalities:

$$
x_{k+1}^{a T} P_{\alpha(k+1)}^{a} x_{k+1}^{a}-x_{k}^{a T} P_{\alpha(k)}^{a} x_{k}^{a}-r_{k}^{T} r_{k}+\beta_{i}^{2} f_{k}^{T} f_{k}<0
$$

and with similar steps of calculations as in the proof of Theorem (1), the LMIs in (44a) are the deduced.

On the other hand, in order to guaranty the stability of the observer, the SLF must be positive and decreasing, which is expressed by the following inequality:

$$
x_{k+1}^{a T} P_{\alpha(k+1)}^{a} x_{k+1}^{a}-x_{k}^{a T} P_{\alpha(k)}^{a} x_{k}^{a}<0
$$

which yields to the LMI (44b).

Following the steps 1), 2) and 3) of the algorithm given in section III, the observer gain in step 4) is then computed using numerical optimization tool by maximizing the LMIS in (44) subject to $\beta_{i}$. And finally compute the unknown matrices of the observer as in step 5).

Remark 4: The $P_{\alpha(k)}^{a}$ matrices in the inequality (45) are not required to be sign definite (positive definite), in the sens of the $H_{-}$index for the fault detection.

However, these matrices should be positive definite and this is a necessary condition in order to get positive Switched Lyapunov Functions, and hence proofing the stability of the observer (in fault free case) and the FDO.

\section{EXAMPLE}

Consider the LTI switched MIMO system of the form (1) with the following matrices:

$$
\begin{gathered}
A_{1}=\left[\begin{array}{ccc}
0.96 & 0.04 & 0.02 \\
0.012 & 0.60 & 0.64 \\
-0.06 & 0.08 & 0.94
\end{array}\right], E_{f_{1}}=\left[\begin{array}{c}
0.06 \\
1.2 \\
0.18
\end{array}\right], \\
A_{2}=\left[\begin{array}{ccc}
0.96 & 0.04 & 0.02 \\
0.012 & 0.60 & 0.14 \\
-0.02 & 0.08 & 0.94
\end{array}\right], E_{f_{2}}=\left[\begin{array}{c}
0.07 \\
1.4 \\
0.49
\end{array}\right], \\
C_{1,2}=\left[\begin{array}{ccc}
0.4 & 0 & 0 \\
0 & .12 & 0
\end{array}\right] ; \quad F_{f_{1,2}}=[0.01] .
\end{gathered}
$$

The initial conditions are $x(0)=\left[\begin{array}{lll}1 & 2 & 3\end{array}\right]^{T}$.

The system is observable. A stable $\mathcal{H}_{-}$FD minimal order observer can be designed.

First, define the matrices $E_{i}$, and compute $T_{i}$ and $K_{i}$ :

$$
E_{1}=E_{2}=\left[\begin{array}{ccc}
10 & 10 & 4 \\
-200 & 1 & -10
\end{array}\right] ; \operatorname{rank}\left(\begin{array}{l}
E_{1 \& 2} \\
C_{1 \& 2}
\end{array}\right)=3
$$

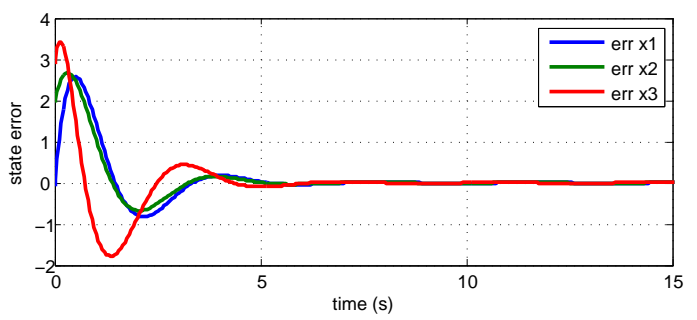

Fig. 2. State estimation errors in fault-free case
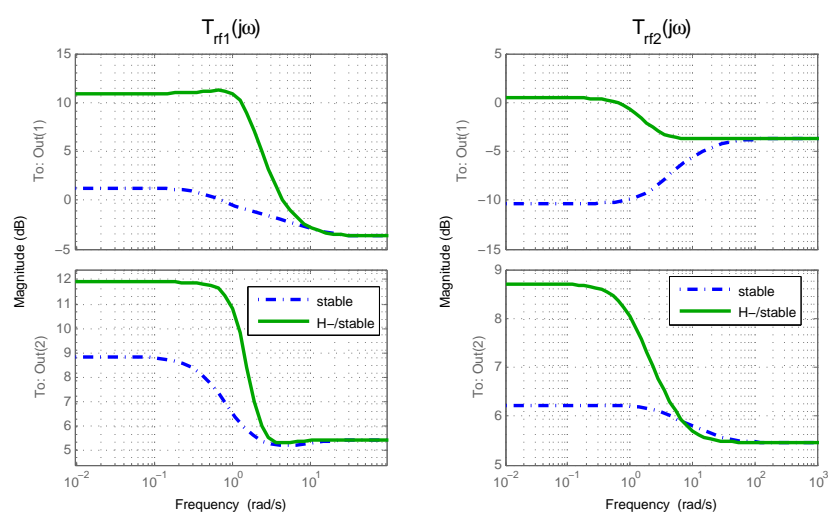

Fig. 3. Sensitivity plots $T_{r f_{1}}(j \omega)$ (right) and $T_{r f_{2}}(j \omega)$ (left)

Then define the loop shaping matrices and weighting filter: suitable $D_{a d d, i}$ matrices are chosen for the additive filter. Let

$$
\begin{gathered}
D_{a d d, 1}=D_{a d d, 2}=\left[\begin{array}{ll}
0.046 & 0.04
\end{array}\right]^{T}, \\
\text { and } \quad F_{H, 1}(s)=F_{H, 2}(s)=\left(\frac{s / .095+1}{s / 8+1}\right)^{2}
\end{gathered}
$$

Using Matlab optimization tools such YALMIP or SeDuMi, the set of LMIs can be numerically solved.

The plots in figure (2) show that the state estimation error converge to zero in the fault free case.

Finally, the faults or residuals sensitivity are plot in figure (3). It could be noticed that the fault sensitivity with the FDO in low frequencies has been enhanced, compared to the simple stability observer design SO. In addition, this was done locally on low frequencies, while noticing that the global minima of the sensitivity functions are located in high frequencies. These results prove the utility of the loop shaping method applied in the proposed method.

\section{CONCLUSION AND FURTHER WORK}

In this paper, LMI conditions have been proposed to design a reduced order state estimator and a fault detection observer for LTI switched systems.

First the condition of existence of the generalized observer are stated and proved. Then two design objectives are formulated using LMIs: 1) stabilize the observer and 2) maximize the $H_{-}$-index from the faults to the residuals. The results are obtained using switched Lyapunov functions in order to avoid conservatism introduced by single quadratic Lyapunov functions.

Possible extensions should concern robust observation, fault tolerant control and robust control. 


\section{APPENDIX}

Denote:

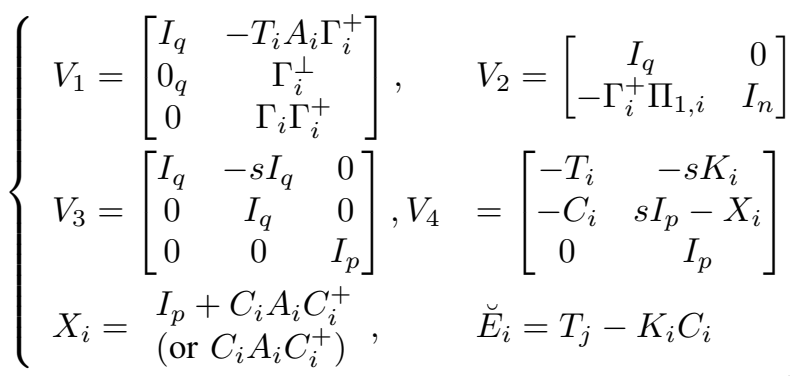

$V_{1}$ is of rank $n+q$ and $V_{4}$ is of rank $n+p$ from (A.2), $V_{2}$ and $V_{3}$ are regular matrices of rank $n+q$ and $p+2 q$ respectively.

Consider two matrices $A: n \times m$ and $B: m \times p$, the Sylvester's inequality states:

$$
\begin{aligned}
\operatorname{rank}(A)+\operatorname{rank}(B)-m & \leq \operatorname{rank}(A B) \\
& \leq \min (\operatorname{rank}(A), \operatorname{rank}(B))
\end{aligned}
$$

Then under assumptions (A.1) and (A.2), the following equalities hold:

$$
\begin{array}{r}
n=\operatorname{rank}\left(\begin{array}{c}
z I_{n}-A_{i} \\
C_{i}
\end{array}\right)=\operatorname{rank}\left(V_{4}\left(\begin{array}{c}
z I_{n}-A_{i} \\
C_{i}
\end{array}\right)\right) \\
=\operatorname{rank}\left(\begin{array}{c}
T_{i} A_{i}-z T_{i}-z K_{i} C_{i} \\
C_{i} A_{i}-X_{i} C_{i} \\
C_{i}
\end{array}\right) \\
=\operatorname{rank}\left(\begin{array}{c}
T_{i} A_{i}-z E_{i} \\
C_{i}(\text { or } 0) \\
C_{i}
\end{array}\right)=\operatorname{rank}\left(\begin{array}{c}
T_{i} A_{i}-z E_{i} \\
C_{i}
\end{array}\right) \quad(49 \mathrm{a}) \\
\operatorname{rank}\left(\begin{array}{cc}
z I_{q} & T_{i} A_{i} \\
I_{q} & E_{i} \\
0 & C_{i}
\end{array}\right)=\operatorname{rank}\left(V_{3}\left[\begin{array}{cc}
z I_{q} & T_{i} A_{i} \\
I_{q} & E_{i} \\
0 & C_{i}
\end{array}\right]\right) \\
=\operatorname{rank}\left(\begin{array}{cc}
0 & T_{i} A_{i}-z E_{i} \\
I_{q} & E \\
0 & C_{i}
\end{array}\right)=\operatorname{rank}\left(\begin{array}{c}
T_{i} A_{i}-z E_{i} \\
C_{i}
\end{array}\right)+q
\end{array}
$$

$$
\begin{array}{r}
\operatorname{rank}\left(\begin{array}{cc}
z I_{q} & T_{i} A_{i} \\
I_{q} & E_{i} \\
0 & C_{i}
\end{array}\right)=\operatorname{rank}\left(V_{1}\left[\begin{array}{cc}
z I_{q} & T_{i} A_{i} \\
I_{q} & E_{i} \\
0 & C_{i}
\end{array}\right] V_{2}\right) \\
=\operatorname{rank}\left(\begin{array}{cc}
z I_{q}-T_{i} A_{i} \Gamma_{i}^{+} \Pi_{1, i} & 0 \\
\Gamma_{i}^{\perp} \Pi_{1, i} & 0 \\
0 & \Gamma_{i}
\end{array}\right) \\
=\operatorname{rank}\left(\begin{array}{c}
z I_{q}-T_{i} A_{i} \Gamma_{i}^{+} \Pi_{1, i} \\
\Gamma_{i}^{\perp} \Pi_{1, i}
\end{array}\right)+n \\
=\operatorname{rank}\left(\begin{array}{c}
z I_{q}-\mathbb{A}_{1, i} \\
\mathbb{A}_{2, i}
\end{array}\right)+n
\end{array}
$$

Finally, using (49a) in (49b), then (49c), the following equivalence holds:

$$
\operatorname{rank}\left(\begin{array}{c}
z I_{n}-A_{i} \\
C_{i}
\end{array}\right)=n \Leftrightarrow \operatorname{rank}\left(\begin{array}{c}
z I_{q}-\mathbb{A}_{1, i} \\
\mathbb{A}_{2, i}
\end{array}\right)=q
$$

\section{REFERENCES}

[1] S. X. Ding, Model-Based Fault Diagnosis Techniques. Springer, 2013.

[2] R. Isermann, "Model-based fault-detection and diagnosis : status and applications," Annual Reviews in Control, vol. 29, pp. 71 - 85, 2005.

[3] H. Inseok, K. Sungwan, K. Youdan, and C. E. Seah, "A survey of fault detection, isolation, and reconfiguration methods," IEEE Transaction on control systems technology, vol. 18, pp. $636-653,2010$.

[4] R. Patton and J. Chen, "Application aspects of robustness for FDI using observer methods," Journal of control Engineering Practice, vol. 5, pp. 671-682, 1997.

[5] D. Liberzon, Switching in Systems and Control. Birkhäuser, 2003.

[6] H. Lin and P. J. Antsaklis, "Stability and stabilizability of switched linear systems: A survey of recent results," IEEE Transactions On Automatic Control, vol. 54, pp. 308-322, 2009.

[7] E. G. Nobrega, M. O. Abdalla, and K. Grigoriadis, "Robust fault estimation of uncertain systems: an lmi-based approach," International Journal of Robust and Nonlinear Control, pp. 1657-1680, 2008.

[8] J. Liu, J. L. Wang, and G.-H. Yang, "An LMI approach to minimun sensitivity analysis with application to fault detection," Automatica, vol. 41, pp. 1995-2004, 2005.

[9] I. M. Jaimoukha, Z. H. Li, and V. Papakos, "A matrix factorization solution to the $h-/ h_{\infty}$ fault detection problem," Automatica, pp. 1907 191, 2006.

[10] J. L. Wang, G.-H. Yang, and J. Liu, "An LMI approach to $\mathcal{H}_{-}$index and mixed $\mathcal{H}_{-} / \mathcal{H}_{\infty}$ fault detection observer design," Automatica, vol. 43, pp. 1656-1665, 2007.

[11] D. Koenig, "Observer design for unknown input nonlinear descriptor systems via convex optimization," IEEE Transactions On Automatic Control, vol. 51, no. 6, pp. 1047-1052, 2006.

[12] A. Alif, M. Darouach, and M. Boutayeb, "Design of robust $H_{\infty}$ reduced-order unknown-input filter for a class of uncertain linear neutral systems," IEEE Transactions On Automatic Control, vol. 55, pp. 6-19, 2010.

[13] C. R. Rao and S. K. Mitra, "Generalized inverse of matrices and its applications," New York: Wiley, pp. 601-620, 1971.

[14] A. Farhat and D. Koenig, " $\mathcal{H}_{-} / \mathcal{H}_{\infty}$ fault detection observer for switched systems," Proceedings of the 53th IEEE Conference on Decision and Control, 2014. 